\title{
Correlación entre la Ecocardiografía Transesofágica Tridimensional y Tomografía Cardíaca en la determinación del Anillo Aórtico previo al Reemplazo de Válvula Aórtica con Catéteres (TAVR)
}

\author{
Rodrigo Ibáñez-Arenas, Gabriel Maluenda, Martín Larico, Eduardo Bórquez, Mario Araya, Marcelo \\ Maulén, Christian Dauvergne, Michael Howard, Rodrigo Castillo, Luis Garrido. \\ Unidad de Ecocardiografía y Hemodinamia. Departamento de Cardiología. Clínica Alemana de \\ Santiago.
}

Introducción: Para el reemplazo de válvula aórtica con catéteres (TAVR) es fundamental la adecuada medición del anillo aórtico para elegir el tamaño adecuado de la prótesis y evitar complicaciones graves como la ruptura del anillo aórtico y la insuficiencia paravalvular (IAP). La tomografía computada cardíaca (TC) es el estándar de oro en la medición del anillo aórtico, pero la ecocardiografía transesofágica 3D (ETE3D) parece ser una alternativa atractiva.

Objetivo: Evaluar la correlación entre las mediciones del anillo aórtico obtenidas por ETE3D y las encontradas en la TC. Los resultados obtenidos se evaluaron en términos de la prótesis elegida y la presencia de insuficiencia aórtica post TAVR.

Métodos: Se realizaron de manera retrospectiva y ciega, las mediciones del anillo aórtico por TC y
ETE3D en 18 pacientes consecutivos, edad promedio $82 \pm 7,06$ años, sometidos a TAVR usando prótesis Sapien XT (Edwards Lifescience). Se comparó el diámetro máximo, diámetro mínimo, diámetro medio, área y perímetro del anillo.

Resultados: La ETE3D subvaloró el tamaño del anillo aórtico, pero demostró tener una buena correlación con los diferentes parámetros de la TC, con mejor comportamiento para el perímetro del anillo. ETE3D y TC coincidieron en la elección de la prótesis en 17 pacientes y no hubo IAP de relevancia.

Conclusión: La ETE3D tiene buena correlación comparada contra la TC en la valoración del anillo aórtico. La ETE3D parece una atractiva alternativa a la TC para medición del anillo especialmente en casos complejos o cuando la disponibilidad y acceso a la TC puede estar limitada.

\section{Correspondencia:}

Dr. Rodrigo Ibáñez Arenas.

Departamento de Cardiología.

Vitacura 5951, Santiago.

Clínica Alemana de Santiago.

ribanez@alemana.cl 


\section{Correlation between 3-D trans-esophageal echocardiography and Cardiac Computed tomography in the evaluation of the aortic annulus prior to Transcatheter aortic Valve implantation (TAVR)}

In TAVR, the precise measuring of the aortic annulus is essential to determine the size of the prosthesis and to avoid complications like rupture of the annulus of aortic insufficiency. Computed tomography (CT) is the gold standard to achieve these goals. 3-D trans-esophageal echocardiography (3-D TEE) to be a useful alternative method.

Aim: to evaluate the correlation of aortic annulus measurements obtained with 3-dTE and CT in the determination of annulus measurements before TAVR. Prosthesis size and presence of aortic insufficiency were related to these measurements.

Methods: In a retrospective blinded protocol the measurements of the aortic annulus obtained with CT or 3D-TEE were correlated in 18 consecutive patients (mean age $82 \pm 7.1$ ) submitted to TAVR with a Sapien XT (Edwards Lifescience) prosthesis. Maximal, mini- mal and mean diameters as well as area and annular circumference were evaluated.

Results: Compared to CT, 3D-TEE under estimated de size of the aortic annulus but correlated well with other measurements of CT, especially with the annular circumference. Measurements from both methods agreed in the determination of the prosthesis size in 17 of 18 patients. No significant per prosthetic aortic insufficiency was observed in any case.

Conclusion: 3DTEE correlates well with $\mathrm{CT}$ in evaluating the different parameters of the aortic annulus and is a good alternative to $\mathrm{CT}$ in complex cases and when $\mathrm{CT}$ is not readily available.

Keywords: Three dimensional echocardiography, aortic valve, multidetector computed tomography, aortic stenosis, transcatheter aortic valve implantation. 


\section{Introducción:}

El reemplazo de válvula aórtica con catéteres (TAVR) está aceptado como alternativa al tratamiento quirúrgico de la estenosis aórtica en pacientes de alto riesgo y es la elección en los casos inoperables. ${ }^{1-5}$ La insuficiencia aórtica paravalvular (IAP) de cualquier grado está presente en las distintas series en más del $80 \%$ de los pacientes sometidos a TAVR y se ha asociado a un aumento de la mortalidad, especialmente cuando esta es moderada o severa, presente en el $7 \%$ al $24 \%$ de los pacientes. ${ }^{3-6-9}$ La principales causas de IAP son la elección de una prótesis más pequeña que el anillo aórtico del paciente y la inadecuada posición del dispositivo, pero existen otros predictores como la excentricidad del anillo y el grado o distribución de calcificaciones de los velos. ${ }^{9-10}$

Múltiples estudios sugieren que la TC, puede realizar la medición más exacta de las dimensiones del anillo aórtico, lo que permite una correcta elección del tamaño de la prótesis a instalar disminuyendo la incidencia de IAP. ${ }^{9-11} \mathrm{Se}$ han desarrollado algoritmos para la elección de la prótesis a instalar, basados en los resultados de la medición del anillo aórtico evaluado por la TC respecto al área, perímetro, diámetro máximo, mínimo y diámetro promedio. ${ }^{11-14}$

La ecocardiografía 2D (superficie y transesofágica) ha sido el método más utilizado para la medición del anillo aórtico, pero existe importante evidencia que indica que subestima los valores reales de este. ${ }^{12-14}$ La ETE3D, menos estandarizada que la TC, podría ser una alternativa para medir en forma certera y reproducible el anillo aórtico y contribuir al monitoreo durante el procedimiento, con excelentes resultados del implante y baja incidencia de IAP. ${ }^{9-14-15}$

\section{Objetivos:}

El objetivo de este estudio fue evaluar la correlación de las mediciones del anillo aórtico por ETE3D y la TC. Secundariamente, se buscó valorar estos resultados en términos de la prótesis elegida y la presencia de IAP post TAVR.

\section{Métodos:}

Desde el año 2010 hasta el año 2014, 40 pacientes con diagnóstico de estenosis aórtica severa sintomática, fueron sometidos a TAVR usando una prótesis expandible con balón, Sapiens XT, Edwards Lifescience ${ }^{\circledR}$, en Clínica Alemana de Santiago y fueron incluidos prospectivamente en una base de datos local. Los pacientes fueron sometidos a una evaluación sistemática, multidisciplinaria con ecocardiografía, coronariografía, angiografía aortofemoral e iliofemoral y TC. El implante fue exitoso en el 100\% de los casos. Todos los pacientes firmaron un consentimiento informado para los procedimientos.

Se seleccionaron los últimos 18 pacientes consecutivos en los cuales se dispuso de ETE3D durante el procedimiento. En estos pacientes se realizaron de manera retrospectiva y ciega las mediciones del anillo aórtico utilizando las imágenes obtenidas por ETE3D y TC: diámetro máximo, diámetro mínimo, diámetro medio, área y perímetro del anillo. Para cada modalidad, la adquisición de imágenes y las mediciones fueron realizadas por 1 médico especialista en cada técnica.

Ecocardiografía Transesofágica 3D: Fue realizada con un sistema de ecocardiografía Vivid E9, con un transductor transesofágico 4D 6T (General Electric Systems $\left.{ }^{\circledR}\right)$ que permite realizar ETE3D en vivo, imágenes ortogonales en 3 planos, volumen completo 3D gatillado, volumen completo 3D con Color y ecocardiografía convencional 2D en múltiples planos, con color y Doppler espectral. Las imágenes fueron adquiridas en volumen completo 3D en un único latido. Se realizaron mediciones del anillo aórtico en vivo que fueron utilizadas para la toma de decisiones durante el TAVR, obteniendo una imagen transesofágica 2D en eje largo apical a 130 grados aproximadamente, visualizando el tracto de salida del ventrículo izquierdo y la aorta ascendente; se activó el modo $4 \mathrm{D}$ en volumen completo para adquirir un video de un latido, con más de 10 cuadros/seg. Se procesó el volumen completo en el programa 4D del equipo, que permite obtener los planos ortogonales sagital, coronal y transverso. Todos los planos fueron alineados por el centro de la válvula aórtica abierta, en sístole, con el plano sagital y coronal ubicados en forma paralela al eje largo de la aorta ascendente. Los planos ortogonales fueron rotados para identificar los puntos de anclaje más caudales de los velos aórticos y el plano transverso fue desplazado hacia el ventrículo izquierdo hasta llegar a los puntos de inserción de los velos aórticos (Fig 1). En forma retrospectiva y ciega, en la estación de trabajo EchoPac, General Electric Systems ${ }^{\circledR}$, se realizaron las mediciones del anillo aórtico, que fueron las utilizadas para efectos de este estudio.

La cuantificación de la insuficiencia aórtica se realizó según los criterios ecocardiográficos estandarizados, previamente descritos. ${ }^{16}$

Paso 1, se muestra la creación del plano transverso a partir de los planos ortogonal 1 (verde) y 2 (amarillo), que deben estar paralelos a la unión sinotubular de la aorta. Paso 2, se gira el eje ortogonal 1 (verde) sobre el plano transverso, 


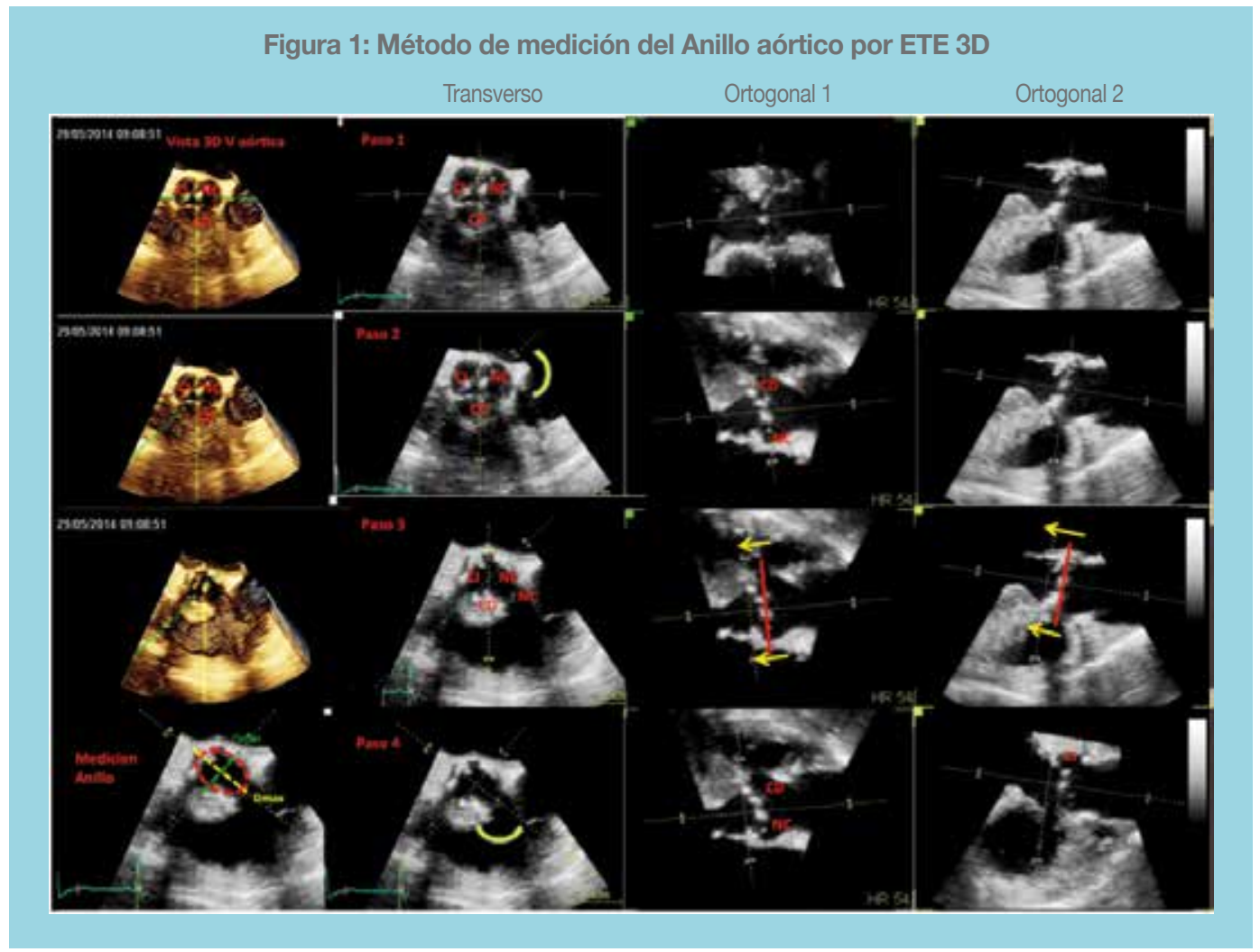

para lograr identificar la unión más caudal del velo coronariano derecho (CD) y el no coronariano (NC). Paso 3, el plano transverso (blanco) se mueve en dirección caudal en los ejes ortogonales 1 y 2 , hasta los puntos de inserción de los velos CD y NC. Paso 4, se rota el eje ortogonal 2 (amarillo) para alinear el punto caudal de inserción del velo coronariano izquierdo (CI). Luego se traza el perímetro y área (rojo), diámetro máximo (Dmax) y diámetro mínimo (Dmin) del anillo aórtico.

Tomografía computada cardíaca: Todos los pacientes sometidos a TAVR fueron estudiados con en un equipo Siemens multicorte de 128 canales. Se obtuvieron imágenes gatilladas cardíacas y de aorta torácica, tras lo cual se realizó "barrido" tomográfico de aorta abdominal y sistema iliofemoral. Se determinaron las dimensiones del anillo aórtico incluyendo diámetro mayor y menor, área y perímetro; altura de nacimiento de las coronarias, dimensiones de los senos de Valsalva y la unión sinotubular. (Figura 2) Se determinaron los diámetros menores en los segmentos ilíacos y femorales; se valoró el grado de calcificación y tortuosidad de los accesos para elegir la mejor vía para el TAVR.

Procedimiento: El TAVR fue realizado en la sala de cate-
Figura 2: Dimensiones de anillo aórtico obtenidas por tomografía cardíaca
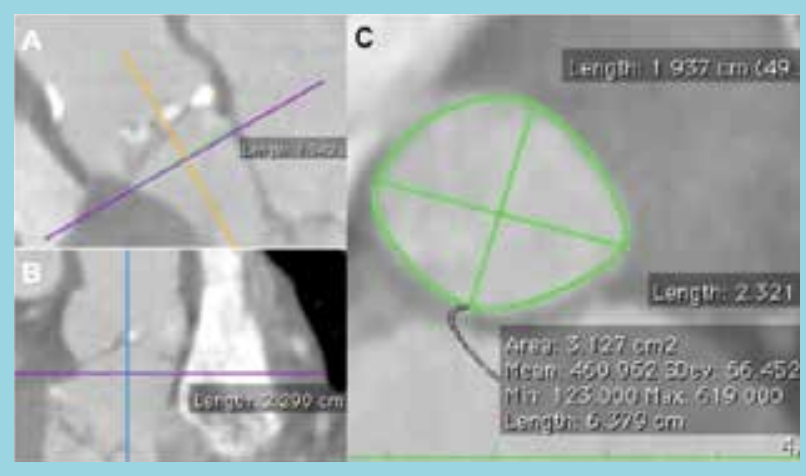

Diámetro en plano sagital (A). Diámetro en plano coronal (B). Diámetros, área y perímetro obtenidos en plano axial $(C)$.

terismo cardíaco provista de todas las capacidades angiográficas y de un quirófano; incluyendo respaldo de perfusión y circulación extracorpórea. Todos los casos fueron realizados bajo intubación y anestesia general. El acceso a la arteria femoral fue realizado por punción o exposición quirúrgica, utilizando un introductor eSheath (Edwards Lifescience, Irvine, CA, USA) expandible de 16 a 20-Fr que fue avanzado sobre una guía rígida. Se administró Heparina (70 unidades/Kg) para obtención y mantención 
de un tiempo de coagulación activada $>250$ segundos. Se procedió a cruzar la válvula aórtica y a realizar una pre dilatación con balón. El arco aórtico fue navegado con un sistema de deflexión NovaFlex (Edwards Lifescience). Se realizó el implante de la prótesis balón expandible Sapien XT (Edwards Lifescience) de 23, 26 o 29 mm según las dimensiones del anillo aórtico, bajo estimulación ventricular derecha a alta frecuencia (180-200 lpm) con técnica estándar. Para el acceso transapical, se realizó abordaje quirúrgico vía minitoracotomía lateral izquierda a nivel apical. Tras la preparación del ápex con doble sutura del miocardio en "jareta", se puncionó con aguja de 18-gauge. Se cruzó la válvula aórtica y se avanzó un introductor con sistema de liberación Ascendra-II ${ }^{\mathrm{TM}}$ (Edwards Lifescience) de 24-26-Fr sobre guía rígida. Luego de realizar valvuloplastía aórtica, se implantó una prótesis Sapien XT de 23, 26 o 29 mm según las dimensiones del anillo aórtico, bajo estimulación ventricular derecha a alta frecuencia (180-200 lpm) con técnica estándar.

Elección del tamaño de la prótesis: Se hizo según las recomendaciones existentes, basados en las dimensiones obtenidas previas al procedimiento por TC, utilizando especialmente los algoritmos que consideran el área y perímetro del anillo. En los casos en que las dimensiones del anillo fueron limítrofes y se planteaba la duda entre dos diferentes tamaños de prótesis, se realizó valvuloplastía aórtica con balón para seleccionar el tamaño del dispositivo, como ha sido descrito. ${ }^{11-14}$

Análisis estadísticos: Las variables continuas fueron presentadas como media \pm desviación estándar (DE) y las variables categóricas como valor absoluto y porcentajes. Las diferencias entre variables continuas fueron evaluadas por test " $t$ " de Student. La estimación de la interdependencia entre las dimensiones del anillo medidas por la TC y ETE3D se realizó empleando el Coeficiente de correlación de Pearson, y además, considerando la existencia de mediciones de las variables en estudio sin distribución normal, se hizo también el análisis de Correlación de Spearman.

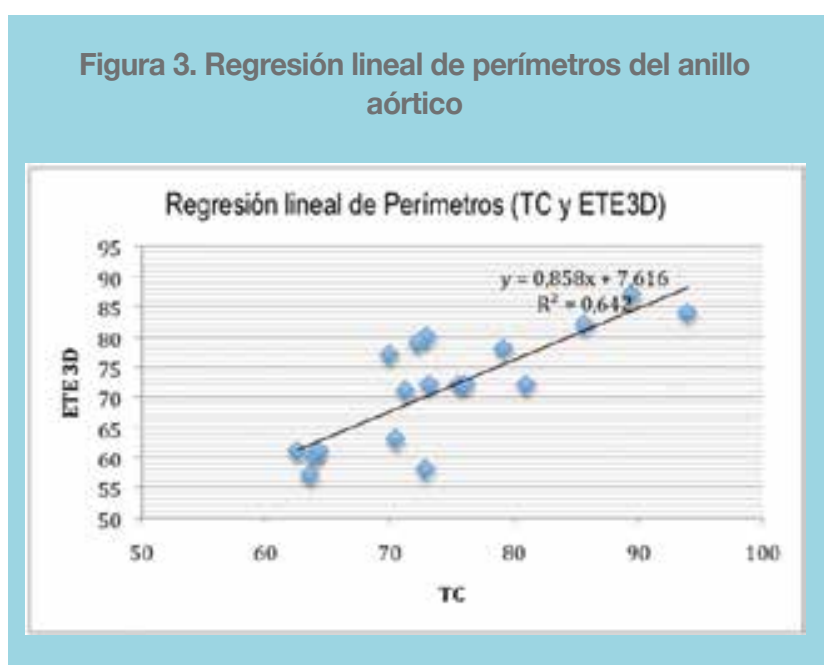

\section{Resultados:}

Se estudiaron 18 pacientes consecutivos sometidos a TAVR, edad promedio $82 \pm 7,06$ años y 7 (39\%) mujeres. 13 pacientes recibieron TAVR transfemoral $(72,2 \%), 5$ pacientes vía transapical y 4 pacientes recibieron TAVR en válvula bicúspide. La fracción de eyección promedio del ventrículo izquierdo fue $61,4 \pm 11,1$ (\%). Se implantaron 7 prótesis No 23, 7 No 26 y 4 No 29.

Respecto a la IAP, $14(77.8 \%)$ pacientes tuvieron grado leve $(1 / 4)$ y $4(22,2 \%)$ grado leve a moderado $(2 / 4)$.

Las dimensiones del anillo aórtico obtenidas por ETE3D y TC son presentadas en la Tabla 1. Al comparar ambos métodos se observó que la ETE3D tiende a subestimar las mediciones del anillo, aunque la ETE3D demostró tener una muy buena correlación con los diferentes parámetros obtenidos por TC, con el mejor comportamiento para el perímetro del anillo (Tabla 1, Figura 3).

ETE3D y TC coincidieron en la elección de la prótesis en 17 pacientes. En 1 caso con válvula bicúspide, la elección de la prótesis favoreció al ETE3D. La selección final de la prótesis se basó en la evaluación con valvuloplastía aórtica con balón.

\begin{tabular}{|l|c|c|c|c|c|}
\multicolumn{7}{|c|}{ Tabla 1. Correlación parámetros de TC y ETE3D del anillo aórtico } \\
\hline & TAC (mm o mm2) & ECO 3D (mm o mm2) & C. Spearman & r de Pearson & $p$ \\
\hline Área & $431,17 \pm 97,84$ & $386,67 \pm 99,94$ & 0,863 & 0,861 & $<0,01$ \\
\hline Perímetro & $74,38 \pm 8,85$ & $71,44 \pm 9,48$ & 0,777 & 0,801 & $<0,05$ \\
\hline Diámetro máximo & $26,29 \pm 2,99$ & $24,67 \pm 2,72$ & 0,794 & 0,772 & $<0,01$ \\
\hline Diámetro mínimo & $20,93 \pm 2,90$ & $19,28 \pm 3,27$ & 0,766 & 0,781 & $<0,01$ \\
\hline Diámetro medio & $23,61 \pm 2,69$ & $21,92 \pm 2,92$ & 0,828 & 0,848 & $<0,01$ \\
\hline
\end{tabular}




\section{Discusión:}

La medición del anillo aórtico es fundamental para la adecuada elección del tamaño de la prótesis para TAVR. La ecocardiografía transesofágica 2D está recomendada y es la técnica más común para el análisis del anillo aórtico, sin embargo, asume un anillo circular. ${ }^{4-14}$ Varios estudios utilizando TC, resonancia nuclear magnética de corazón y ETE3D han mostrado que la gran mayoría de los anillo aórticos tienen una geometría excéntrica, elipsoide u oval. ${ }^{11-17}$ En nuestro estudio, todos los pacientes estudiados mostraron morfologías del anillo no circulares en las mediciones realizadas por TC y por ETE3D.

En el TAVR, el TC ha sido recomendado para la elección de las prótesis auto expandibles o expandibles con balón, para evitar la desproporción entre el anillo valvular aórtico nativo y la prótesis implantada, con el fin de prevenir la IAP. ${ }^{11-18}$ Actualmente se recomienda una leve sobre dimensión de la prótesis a instalar respecto al área del anillo aórtico nativo para evitar esta complicación. ${ }^{12-14}$

Nuestro estudio, muestra que el ETE3D subestima las mediciones del anillo aórtico respecto al TC, pero se correlaciona de muy buena forma respecto al área, perímetro y diámetro medio ( $\mathrm{r}$ entre 0,77 a 0,86 ) Jilaihawi y cols 21 , reportaron datos similares en 256 pacientes, , pero con coeficientes de correlación menores ( $\mathrm{r}$ entre 0,62 a 0,72 ). Arnold y cols 22, en 53 pacientes, encontraron que el área del anillo medida por ETE3D era subestimada en un 9\% respecto a la TC. Nosotros encontramos un $10 \%$ de sub estimación en el área, 9,6\% en el perímetro y un 7\% respecto al diámetro medio.

En 17 de los casos evaluados no hubo discrepancias entre el ETE3D y la TC respecto a la elección de la prótesis a implantar. Sólo en un paciente portador de una válvula aórtica bicúspide con anillo de morfología oval, las mediciones obtenidas por el ETE3D sugerían elegir una prótesis N0 $29 \mathrm{~mm}$ y la TC determinaba dimensiones limítrofes para una prótesis N0 26 o $29 \mathrm{~mm}$. Para aclarar esta diferencia se decidió realizar una evaluación del anillo aórtico con balón de plastía aórtica durante la cineangiografía, ${ }^{6}$ que finalmente determinó implantar la prótesis $\mathrm{N0} 29 \mathrm{~mm}$; el resultado del implante fue exitoso, quedando con una IAP leve. Otros 3 pacientes recibieron TAVR en válvula bicúspide y no hubo discrepancia en la elección de la prótesis entre el ETE3D y la TC. Si bien es cierto, el implante TAVR en válvula bicúspide es complejo y menos frecuente, existen varios reportes que demuestran que es factible, pero que requiere de una estricta evaluación de la anatomía con el uso de múltiples técnicas de imágenes. ${ }^{20}$

Sólo 4 pacientes $(22,2 \%)$, uno de los cuales fue un implante en válvula aórtica nativa bicúspide, presentaron una IAP grado 2/4 (leve a moderada), lo que está de acuerdo con lo descrito en la literatura usando múltiples técnicas para la elección de la prótesis adecuada para el implante. ${ }^{14-19}$ Este es el primer reporte publicado que realiza de una manera estandarizada las mediciones del anillo aórtico utilizando un equipo de ecocardiografía 3D, Vivid E9, con un transductor transesofágico 4D 6T y su EcoPac (General Electric Systems $\left.{ }^{\circledR}\right)$. Todos los estudios previos han sido realizados en equipos 3D, 3DQ, QLAB; Philips Medical Systems y no en todos los trabajos se detalla el protocolo de reconstrucción multiplanar realizado. El método aquí utilizado es similar al publicado por Kasel y cols 14, pero en nuestro caso, el eje largo de tres cámaras apical obtenido por ecocardiografía transesofágica 2D (aproximado en 130 grados) fue la imagen fundamental para la adquisición del volumen $4 \mathrm{D}$ y realizar la reconstrucción multiplanar del plano transverso. Las razones para elegir este plano es que asegura que las porciones proximales de la aorta y del tracto de salida del ventrículo izquierdo serán incluidas en la adquisición de volumen 4D, para una mejor evaluación del anillo aórtico.

Limitaciones: La posición del transductor transesofágico en cada paciente es variable, por lo tanto los planos sagitales y coronales obtenidos por ETE3D son diferentes a los que se obtienen por TC, las limitaciones de la ventana acústica son inherentes a la ecocardiografía y la medición del anillo es más compleja mientras mayor es el grado de calcificación del anillo. Los datos analizados fueron obtenidos de manera retrospectiva y el número de casos de este estudio es pequeño comparado con los trabajos publicados anteriormente. No fueron evaluadas las diferencias intraobservador e interobservador.

\section{Conclusiones:}

La ETE3D tiene buena correlación comparada con la TC en la valoración del anillo aórtico, aunque esta tiende a subestimar las dimensiones del anillo. Los parámetros del anillo aórtico medido por ETE3D o TC coinciden en la elección de la prótesis. La ETE3D parece una atractiva alternativa a la TC para medición del anillo especialmente en casos complejos o cuando la disponibilidad y acceso a la TC puede estar limitada. 


\section{Referencias}

1. CRIBIER A1, ELTCHANINOFF H, TRON C, BAUER F, AGATIELLO C, SEBAGH L, et al. Early experience with percutaneous transcatheter implantation of heart valve prosthesis for the treatment of end-stage inoperable patients with calcific aortic stenosis. J Am Coll Cardiol 2004; 43: 698-703.

2. GRUBE E, LABORDE JC, GERCKENS U, FELDERHOFF T, SAUREN B, BUELLESFELD L, et al. Percutaneous implantation of the CoreValve self-expanding valve prosthesis in high-risk patients with aortic valve disease: the Siegburg firstin-man study. Circulation 2006; 114: 1616-1624.

3. LEON MB, SMITH CR, MACK M, MILLER DC, MOSES JW, SVENSSON LG, et al; PARTNER Trial Investigators. Transcatheter aortic-valve implantation for aortic stenosis in patients who cannot undergo surgery. N Engl J Med 2010; 363 : 1597-1607.

4. VAHANIAN A, ALFIERI O, AL-ATTAR N, ANTUNES M, BAX J, CORMIER B, et al; European Association of Cardio-Thoracic Surgery, European Society of Cardiology, European Association of Percutaneous Cardiovascular Interventions. Transcatheter valve implantation for patients with aortic stenosis: a position statement from the European Association of Cardio-Thoracic Surgery (EACTS) and the European Society of Cardiology (ESC), in collaboration with the European Association of Percutaneous Cardiovascular Interventions (EAPCI). Eur Heart J 2008; 29: 1463-1470.

5. SMITH C, LEON M, MACK M, MILLER D, MOSES J, SVENSSON L, et al. PARTNER Trial Investigators. Transcatheter versus Surgical Aortic-Valve Replacement in High-Risk Patients N Engl J Med 2011; 364: 2187-2198.

6. CRIBIER A, ELTCHANINOFF H, TRON C, BAUER F, AGATIELLO C, NERCOLINI D, et al. Treatment of calcific aortic stenosis with the percutaneous heart valve: mid-term follow-up from the initial feasibility studies: the French experience. J Am Coll Cardiol 2006; 47: 1214-1223.

7. KODALI SK, WILLIAMS MR, SMITH CR, SVENSSON G, WEBB J, MAKKAR R, et al. Two-year outcomes after transca- theter or surgical aortic-valve replacement. N Engl J Med 2012; 366: $1686-1695$.

8. HAYASHIDA K, LEFÈVRE T, CHEVALIER B, HOVASSE T, ROMANO M, GAROT P, et al. Impact of Post-Procedural Aortic Regurgitation on Mortality After Transcatheter Aortic Valve Implantation. J Am Coll Cardiol Intv 2012; 5: 1247-1256.

9. HAHN RT, KHALIQUE O, WILLIAMS MR, KOSS E, PARADIS JM, DANEAULT B, et al. Predicting Paravalvular Regurgitation Following Transcatheter Valve Replacement: Utility of a Novel Method for Three-Dimensional Echocardiographic Measurements of the Aortic Annulus. J Am Soc Echocardiogr 2013; 26: 1043-1052.

10. ATHAPPAN G, PATVARDHAN E, TUZCU EM, SVENSSON LG, LEMOS PA, FRACCARO C, et al. Incidence, predictors, and outcomes of aortic regurgitation after transcatheter aortic valve replacement: meta-analysis and systematic review of literature. J Am Coll Cardiol 2013;61: 1585-1595.

11. WILLSON AB, WEBB JG, et al. 3-Dimensional aortic annular assessment by multidetector computed tomography predicts moderate or severe paravalvular regurgitation after transcatheter aortic valve replacement: a multicenter retrospective analysis. J Am Coll Cardiol 2012; 59: 1287-1294.

12. WILLSON A, WEBB G, FREEMAN M, WOOD D, GURVITCH R, THOMPSON C, et al. Computed tomography-based sizing recommendations for transcatheter aortic valve replacement with balloon-expandable valves: Comparison with transesophageal echocardiography and rationale for implementation in a prospective trial. Journal of Cardiovascular Computed Tomography 2012; 6: 406-414.

13. GURVITCH R, WEBB J, YUAN R, JOHNSON M, HAGUE C, WILLSON A, et al. Aortic Annulus Diameter Determination by Multidetector Computed Tomography. Reproducibility, Applicability, and Implications for Transcatheter Aortic Valve Implantation J Am Coll Cardiol Intv 2011; 4: 1235-1245.

14. KASEL AM, CASSESE S, BLEIZIFFER S, AMAKI M, HAHN 
RT, KASTRATI A, et al. Standardized Imaging for Aortic Annular Sizing Implications for Transcatheter Valve Selection. J Am Coll Cardiol Img 2013;6:249 - 262.

15. SMITH LA, DWORAKOWSKI R, BHAN A, DELITHANASIS I, HANCOCK J, MACCARTHY PA, et al. Real-Time Three-Dimensional Transesophageal Echocardiography Adds Value to Transcatheter Aortic Valve Implantation. J Am Soc Echocardiogr 2013; 26: 359-369

16. KAPPETEIN A, HEAD S, GE’NE’REUX P, PIAZZA N, VAN MIEGHEM M, BLACKSTONE E, et al. Updated standardized endpoint definitions for transcatheter aortic valve implantation: the Valve Academic Research Consortium-2 consensus document. European Heart Journal 2012; 33: 2403-2418.

17. ALTIOK E1, KOOS R, SCHRÖDER J, BREHMER K, HAMADA S, BECKER M, et al. Comparison of two-dimensional and three-dimensional imaging techniques for measurement of aortic annulus diameters before transcatheter aortic valve implantation. Heart 2011; 97: 1578-84.

18. DELGADO V, NG A, VAN DE VEIRE N, VAN DER KLEY F, SCHUIJF J, LAURENS TOPS L, et al. Transcatheter aortic valve implantation: role of multi-detector row computed tomo- graphy to evaluate prosthesis positioning and deployment in relation to valve function. European Heart Journal 2010; 31: $1114-1123$

19. BLOOMFIELD GS, GILLAM LD, HAHN RT, KAPADIA S, LEIPSIC J, LERAKIS S, et al. A Practical Guide to Multimodality Imaging of Transcatheter Aortic Valve Replacement. J Am Coll Cardiol Img 2012; 5: 441-55.

20. MALUENDA G, ARAYA M, IBAÑEZ-ARENAS R. Successful Transfemoral Aortic Valve Replacement in a Bicuspid Aortic Stenotic Valve: Requirements for a Safe Implant. Catheterization and Cardiovascular Interventions 2013; 82:E826-E830.

21. HASAN JILAIHAWI, MD, NIRAJ DOCTOR, et al. Aortic Annular Sizing for Transcatheter Aortic Valve Replacement Using Cross-Sectional 3-Dimensional Transesophageal Echocardiography. J Am Coll Cardiol 2013; 61: 908-16.

22. ARNOLD NG, DELGADO V, VAN DER KLEY F, SHANKS M, VAN DE VEIRE N, BERTINI M, et al. Comparison of Aortic Root Dimensions and Geometries Before and After Transcatheter and Multislice Computed Tomography Aortic Valve Implantation by 2- and 3-Dimensional Transesophageal Echocardiography. Circ Cardiovasc Imaging. 2010; 3: 94-102. 